\title{
石墨烯和硼氮类石墨烯包覆对 $\mathrm{LiFePO}_{4}$ 表面结构的改进及其电导的 促进作用
}

\author{
孙超严六明*岳宝华 \\ (上海大学理学院化学系, 上海 200444)
}

\begin{abstract}
摘要: 利用密度泛函理论研究石墨烯和硼氮类石墨烯包覆对 $\mathrm{LiFePO}_{4}(010)$ 表面结构和电导性质的影响. 结 果表明包覆层和 $\mathrm{LiFePO}_{4}(010)$ 表面之间的相互作用可以改善 $\mathrm{LiFePO}_{4}(010)$ 表面的电导性能. 石墨烯包覆 $\mathrm{LiFePO}_{4}(010)$ 表面后, 禁带宽度从 $3.3 \mathrm{eV}$ 减小到 $2.1 \mathrm{eV}$. 嗍氮类石墨烯包覆 $\mathrm{LiFePO}_{4}(010)$ 表面后, 虽然其价 带顶和导带底仍由 $\mathrm{Fe}$ 的 $3 d$ 轨道贡献, 但禁带中出现两个间隔为 $0.6 \mathrm{eV}$ 的带隙态, 由嗍氮类石墨烯与 $\mathrm{LiFePO}_{4}$ (010)表面有直接接触并形成共价键的 B 原子和 $N$ 原子贡献.
\end{abstract}

关键词： $\mathrm{LiFePO}_{4}$; 石墨烯; 硼氮类石墨烯; 态密度; 密度泛函理论

中图分类号: 0641; 0649

\section{Improvement of Surface Structure and Enhancement of Conductivity of LiFePO ${ }_{4}$ Surface by Graphene and Graphene-Like B-C-N Coating}

\author{
SUN Chao YAN Liu-Ming* YUE Bao-Hua \\ (Department of Chemistry, College of Sciences, Shanghai University, Shanghai 200444, P. R. China)
}

\begin{abstract}
Density functional theory calculations are used to investigate the surface structure and electric conductivity of the (010) surface of $\mathrm{LiFePO}_{4}$ coated with graphene or graphene-like B-C-N. The calculations indicate that the interaction between the coating and $\mathrm{LiFePO}_{4}(010)$ surface improves the electric conductivity of the $\mathrm{LiFePO}_{4}(010)$ surface. The band gap decreases from 3.3 to $2.1 \mathrm{eV}$ when the $\mathrm{LiFePO}_{4}$ (010) surface is coated with graphene. When the $\mathrm{LiFePO}_{4}(010)$ surface is coated with graphene-like B-C-N, the valence band maximum and conduction band minimum are still dominated by Fe-3d orbitals; however, two in-gap states with an interval of $0.6 \mathrm{eV}$ appear in the band gap, which are attributed to the bonding interaction between graphene-like $\mathrm{B}-\mathrm{C}-\mathrm{N}$ and the $\mathrm{LiFePO}_{4}(010)$ surface.
\end{abstract}

Key Words: $\mathrm{LiFePO}_{4}$; Graphene; Graphene-like B-C-N; Density of states; Density functional theory

\section{1 引 言}

正交橄榄石结构的 $\mathrm{LiFePO}_{4}$ 是一种具有广泛用 途的锂离子电池正极材料, 不仅具有相对于金属锂 为 $3.5 \mathrm{~V}$ 的电极电势和高达 $170 \mathrm{mAh} \cdot \mathrm{g}^{-1}$ 的理论比 容量, 还具有价格低廉、环境友好、化学性质稳定等
优点. ${ }^{1}$ 但是, $\mathrm{LiFePO}_{4}$ 较低的 $\mathrm{Li}^{+}$迁移率和电子电导 率 $\left(298 \mathrm{~K} \text { 下的本征电导率约 } 10^{-9} \mathrm{~S} \cdot \mathrm{cm}^{-1}\right)^{2}$ 制约了它 在动力电池等领域的广泛应用.

在实际工作中, 一般采取降低颗粒粒径和表面 碳包覆等手段改善 $\mathrm{LiFePO}_{4}$ 的电化学性质., ${ }^{3,4}$ 前者可

Received: January 29, 2013; Revised: April 22, 2013; Published on Web: April 23, 2013.

"Corresponding author. Email: liuming.yan@shu.edu.cn; Tel/Fax: +86-21-66132405.

The project was supported by the National Natural Science Foundation of China (21073118), Innovation Program of Shanghai Municipal Education Commission, China (13ZZ078), Shanghai Leading Academic Discipline Project, China (J50101), and Shanghai Higher Education Connotation Construction “085” Project “Materials Genome Engineering”, China.

国家自然科学基金项目(21073118), 上海市教育委员会科研创新项目(13ZZ078), 上海市重点学科计划(J50101)和上海市高等教育内涵建设 “085”工程《材料基因工程》项目资助 
以缩短 $\mathrm{Li}^{+}$和电子在 $\mathrm{LiFePO}_{4}$ 颗粒中的迁移距离; 后 者可以增强 $\mathrm{LiFePO}_{4}$ 颗粒的表面电导率. 此外, Goodenough 等 5 发现在 $\mathrm{LiFePO}_{4}$ 中添加约 $1 \%$ 碳后, $80^{\circ} \mathrm{C} 、 1 C$ 充放电倍率下的实际比容量可达到 160 $\mathrm{mAh} \cdot \mathrm{g}^{-1}$, 有利于提高实际比容量. 不仅如此, 其它 材料也可应用于 $\mathrm{LiFePO}_{4}$ 的包覆改性, 如石墨烯包 覆能改善 $\mathrm{LiFePO}_{4}$ 的电化学性能; ${ }^{6}$ 用聚吡咯包覆花 瓣形 $\mathrm{LiFePO}_{4}$ 颗粒, 充放电倍率和循环性能优于碳 包覆 ${ }^{7}$ 用聚 (3,4-乙撑二氧噻吩)包覆 $\mathrm{LiFePO}_{4}$ 颗粒, $10 C$ 倍率时比容量可达 $123 \mathrm{mAh} \cdot \mathrm{g}^{-1}$. 然而, 降低 $\mathrm{LiFePO}_{4}$ 颗粒粒径和提高包覆物含量都将引起其振 实密度的降低, 不利于能量密度的提高. 因此, 研究 包覆在改善 $\mathrm{LiFePO}_{4}$ 性能中的作用机理, 对进一步 优化 $\mathrm{LiFePO}_{4}$ 的合成工艺和方法具有重要意义. ${ }^{9} 10$

$\mathrm{LiFePO}_{4}$ 的理论和实验研究证明, $\mathrm{Li}^{+}$倾向于沿 着 $\mathrm{LiFePO}_{4}$ 晶胞 $b$ 轴方向一维迁移, 活化能约为 0.6 $\mathrm{eV}^{11,12}$ Fisher 和 Islam $^{13}$ 通过优化 19 个低指数晶面和 计算表面能证明, 无论是热力学控制还是动力学控 制生成的 $\mathrm{LiFePO}_{4}$ 晶体, 皆(010) 晶面占优势, 与 Wang 等 ${ }^{14}$ 的计算结果相同. 水热法合成得到的平板 形 $\mathrm{LiFePO}_{4}$ 颗粒, ${ }^{15,16}$ 大部分暴露面为 $(010)$ 晶面. ${ }^{17}$ 虽 然, 透射电子显微镜观察表明, 碳包覆可以使得 $\mathrm{LiFePO}_{4}$ 表面更加有序; ${ }^{18}$ 但目前仍无法在原子尺度 直接观察碳和 $\mathrm{LiFePO}_{4}$ 表面之间的相互作用. 因此, 碳包覆如何影响 $\mathrm{LiFePO}_{4}$ 表面的化学环境, $\mathrm{LiFePO}_{4}$ 表面的化学环境又如何影响 $\mathrm{LiFePO}_{4}$ 的表面电化学 性质, 以及如何通过改变包覆层的化学组成进一步 提高 $\mathrm{LiFePO}_{4}$ 的电化学性能, 仍然需要进一步探讨 研究.

本文使用密度泛函理论(DFT) 研究 $\mathrm{LiFePO}_{4}$ (010)表面, 比较了无包覆、石墨烯包覆、硼氮类石墨 烯包覆等三种不同的 $\mathrm{LiFePO}_{4}(010)$ 表面结构、能带 结构. 首次提出用硼氮类石墨烯作为 $\mathrm{LiFePO}_{4}$ 包覆 层的设想, 这是 $\mathrm{LiFePO}_{4}$ 包覆改性的研究新方向. 本 文还计算了三种表面结构的态密度(DOS), 探讨了 $\mathrm{DOS}$ 与 $\mathrm{LiFePO}_{4}$ 表面电化学性能的关系, 阐述了 $\mathrm{LiFePO}_{4}$ 碳包覆的作用机理.

\section{2 计算与模型方法}

橄榄石结构的 $\mathrm{LiFePO}_{4}$ 属正交晶系, 具 Pnma 空 间群, 晶胞单元包含四个 $\mathrm{LiFePO}_{4}$ 单位. 其中, $\mathrm{O}$ 原子 以六方密堆积排列, 分别占据两个 $4 \mathrm{c}$ 位 $(\mathrm{O} 1, \mathrm{O} 2)$ 和 一个 $8 \mathrm{~d}$ 位 $(\mathrm{O} 3) . \mathrm{P}$ 原子占据六方密堆积的四面体空
隙形成 $\mathrm{PO}_{4}$ 四面体; $\mathrm{Li}$ 原子占据八面体空隙, 形成 $\mathrm{LiO}_{6}$ 八面体沿 $b$ 轴链状排列; $\mathrm{Fe}$ 原子占据八面体空 隙, 形成 $\mathrm{FeO}_{6}$ 八面体成锯齿状交叉排列(图 1). 这样, 每个 $\mathrm{PO}_{4}$ 四面体的周围有四个 $\mathrm{FeO}_{6}$ 八面体, 其中三 个 $\mathrm{FeO}_{6}$ 八面体和 $\mathrm{PO}_{4}$ 四面体共用角, 一个 $\mathrm{FeO}_{6}$ 八面 体和 $\mathrm{PO}_{4}$ 四面体共用边.

本文选用符合化学计量比的 $\mathrm{LiFePO}_{4}(010)$ 表 面作为研究对象. 一方面, (010)表面是 $\mathrm{Li}^{+}$的迁入和 迁出面; 另一方面, 符合 $\mathrm{LiFePO}_{4}$ 化学计量比的(010) 晶面截取表面能较小, 还能保证 $\mathrm{PO}_{4}$ 四面体的完整. 在表面计算中, 采用由中间层、表面层和真空层构 成的薄片模型(slab model)近似实际体系. 薄片模型 的中间原子被固定, 称为中间层, 用于模拟晶体内 部的周期性环境; 表面原子可以随表面环境的变化 而改变, 称为表面层, 用于模拟表面重构; 真空层用 于屏蔽薄片与薄片之间的相互作用, 构成虚拟的周 期性结构. 在计算中, 根据收玫性测试确定中间层、 表面层、真空层的厚度分别为 $0.58 、 0.32 、 1.00 \mathrm{~nm}$.

在薄片模型中, $b$ 轴方向的晶体周期性结构消 失, $\mathrm{LiFePO}_{4}(010)$ 表面氧原子 $\mathrm{O} 3$ 的部分 $\mathrm{Fe}-\mathrm{O}$ 配位 键被切断, 只剩下一个 $\mathrm{Fe}-\mathrm{O}$ 配位键和一个 $\mathrm{P}-\mathrm{O}$ 共价键. 在距这两个氧原子 $0.15 \mathrm{~nm}$ 的下方有一个 垂直于 $b$ 轴的平面, 有两个 $\mathrm{Fe}$ 原子、两个 $\mathrm{P}$ 原子、四 个 $\mathrm{O}$ 原子; 该平面上的 $\mathrm{Fe}$ 原子也由于 $b$ 轴方向的周 期性消失而缺少一个 $\mathrm{Fe}-\mathrm{O}$ 键, 由六配位变为五配 位, 但两个 $\mathrm{PO}_{4}$ 四面体完整. 石墨烯的结构由包含 16 个 C 原子的石墨烯片段近似表示, 硼氮类石墨烯的 结构由 $\left(\mathrm{BC}_{2} \mathrm{~N}\right)_{4}$ 片段近似表示, 石墨烯片段和嗍氮类 石墨烯片段的悬空键加氢原子使其饱和. $\mathrm{LiFePO}_{4}$ (010)晶面和石墨烯具有不同的周期性, 如计算中采

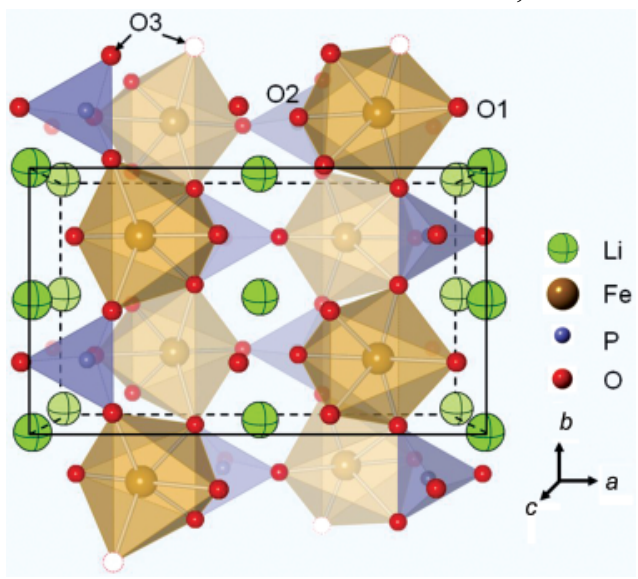

图 $1 \mathrm{LiFePO}_{4}(010)$ 表面的薄片模型的周期性单元

Fig.1 Periodic-unit of the slab model for (010) surface of $\mathrm{LiFePO}_{4}$ 
用连续石墨烯模型, 必将在石墨烯结构中引入应 力, 引起石墨烯的扭曲, 影响计算结果的可靠性. 本 文采用石墨烯片段近似石墨烯, 可克服因 $\mathrm{LiFePO}_{4}$ (010)晶面与石墨烯周期性不匹配对计算结果的影 响. 模型中石墨烯片段长约 $0.706 \mathrm{~nm}$, 晶格长约 $1.043 \mathrm{~nm}$, 相邻石墨烯片段间最短距离约 $0.491 \mathrm{~nm}$, 相互作用已经很弱. 虽然, 构建更大的计算模型可 以更好地近似实际体系, 但计算量太大. 因此, 本文 模型可以在模型的有效性和计算效率之间实现较 好的平衡.

本文 DFT 计算采用 VASP (Vienna $A$-initio Simulation Package)软件, 利用 Hubbard $U$ 修正的广 义梯度近似(GGA)模型. ${ }^{19,20}$ VASP 软件使用赝势近 似原子核和内层电子对外层电子的作用, 使用投影 叠加平面波展开外层电子的波函数. ${ }^{21,22}$ 计算采用 PW91PW91 交换相关泛函和 VWN 插值公式, ${ }^{23,24}$ 使 用 Hubbard $U_{\text {eff }}(U-J)=4.3 \mathrm{eV}$ 修正 Fe 的 $3 d$ 轨道. ${ }^{25}$ 所 有计算开启自旋极化, 平面波截止能量取 $400 \mathrm{eV}$, 以 作用在单个原子上的最大力 $\left(0.1 \mathrm{eV} \cdot \mathrm{nm}^{-1}\right)$ 为收玫标 准. 在优化 $\mathrm{LiFePO}_{4}$ 晶体结构时, 采用 ICSD (Inorganic Crystal Structure Database) 数据库的结构作初始 构型, 使用 $7 \times 9 \times 11$ 的 Monkhorst-Pack $k$ 空间网格, ${ }^{26}$ 保证在倒易空间中网格点之间的间距小于 0.2 $\mathrm{nm}^{-1}$. 在优化表面结构时, 选用优化后的晶体结构参 数作为初始条件, 采用 $k$ 空间 Gamma 点一个格点, 以高斯展开有限温度方法优化表面结构, 以作用在 单个原子上的最大力 $\left(0.5 \mathrm{eV} \cdot \mathrm{nm}^{-1}\right)$ 为收玫标准. 在 计算电荷密度和态密度时, 使用 Blöchl 修正的线性
表 $1 \mathrm{LiFePO}_{4}$ 的理论和实验晶格参数

Table 1 Optimized and experimental lattice parameters of $\mathrm{LiFePO}_{4}$

\begin{tabular}{cccccc}
\hline & $a / \mathrm{nm}$ & $b / \mathrm{nm}$ & $c / \mathrm{nm}$ & $V / \mathrm{nm}^{3}$ & Reference \\
\hline this work & 1.043 & 0.607 & 0.476 & 0.3012 & \\
GGA & 1.039 & 0.604 & 0.473 & 0.2971 & 28 \\
GGA $+U$ & 1.046 & 0.606 & 0.475 & 0.3013 & 27 \\
Expt. & 1.034 & 0.601 & 0.470 & 0.2915 & 29 \\
\hline
\end{tabular}

四面体方法, ${ }^{21}$ 分别在 $3 \times 1 \times 5$ 和 $6 \times 2 \times 10$ 的 $k$ 空间格点 上进行积分. 其它的表面计算参数与晶体结构计算 相同.

\section{3 计算结果与讨论}

\section{1 表面结构}

为了验证研究方法,首先优化了具有周期性边 界条件的 $\mathrm{LiFePO}_{4}$ 晶体, 优化得到的晶胞参数为 $a=$ $1.043 \mathrm{~nm}, b=0.607 \mathrm{~nm}, c=0.476 \mathrm{~nm}$, 体积为 0.3012 $\mathrm{nm}^{3}$. 计算结果与文献报道的结果 ${ }^{27-29}$ 基本一致(表 1), 但比实验结果偏大, 与 GGA 泛函倾向于高估晶 格参数和体积的报道一致. ${ }^{27}$

通过计算薄片模型和体相(bulk)模型的能量差, 可以得到薄片模型的表面能 $(\gamma)$,

$$
\gamma=\frac{E_{\mathrm{s}}-n E_{\mathrm{b}}}{2 A}
$$

其中, $E_{\mathrm{b}}$ 为体相 $\mathrm{LiFePO}_{4}$ 晶胞单元的能量; $E_{\mathrm{s}}$ 为包含 $n$ 个 $\mathrm{LiFePO}_{4}$ 晶胞单元的薄片模型的总能量; $A$ 为薄 片模型的截面积. 计算得到未包覆的 $\mathrm{LiFePO}_{4}(010)$ 表面的表面能为 $0.65 \mathrm{~J} \cdot \mathrm{m}^{-2}$, 与 Wang 等 ${ }^{14}$ 的结果 $\left(0.64 \mathrm{~J} \cdot \mathrm{m}^{-2}\right)$ 一致, 但较 Fisher 等 ${ }^{13}$ 的结果 $\left(0.72 \mathrm{~J} \cdot \mathrm{m}^{-2}\right)$ (a)

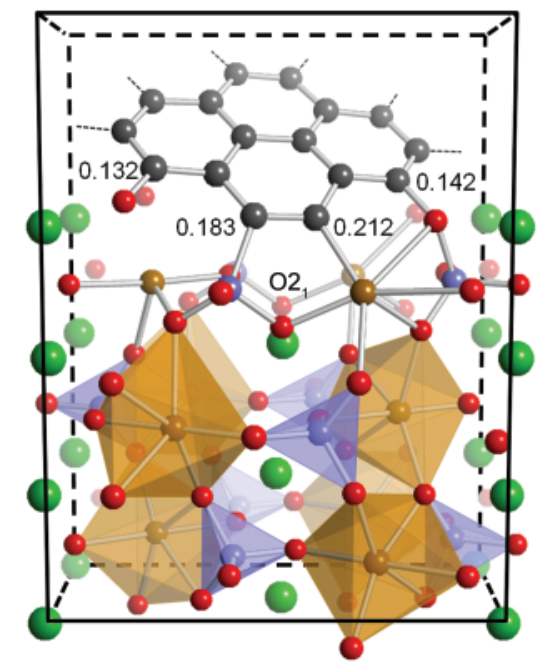

(b)

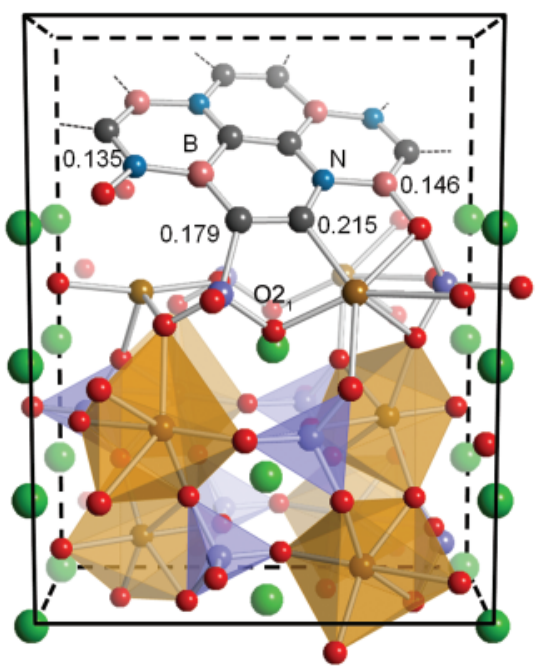

图 2 优化后的石墨烯包覆(a)和硼氮类石墨烯包覆(b)的 $\mathrm{LiFePO}_{4}(010)$ 表面

Fig.2 Optimized (010) surfaces of $\mathrm{LiFePO}_{4}$ coated with graphene (a) and graphene-like B-C-N (b) 
表 $2 \mathrm{LiFePO}_{4}(010)$ 表面原子在 $a, b$ 和 $c$ 轴方向的位移(单位为 $\mathrm{nm}$ )

Table 2 Displacements (in $\mathrm{nm}$ ) of atoms on $\mathrm{LiFePO}_{4}(010)$ surface along lattice axes $a, b, c$

\begin{tabular}{|c|c|c|c|c|c|c|c|c|c|c|}
\hline \multirow{2}{*}{ Atom } & \multirow{2}{*}{$\begin{array}{c}\text { Coordinate } \\
\text { number }\end{array}$} & \multicolumn{3}{|c|}{ Pristine surface } & \multicolumn{3}{|c|}{ Coated with graphene } & \multicolumn{3}{|c|}{ Coated with B-C-N } \\
\hline & & $\Delta a$ & $\Delta b$ & $\Delta c$ & $\Delta a$ & $\Delta b$ & $\Delta c$ & $\Delta a$ & $\Delta b$ & $\Delta c$ \\
\hline $\mathrm{Fe}_{1}$ & 5 & 0.010 & -0.012 & 0.023 & -0.001 & 0.005 & 0.001 & 0.005 & 0.011 & 0.002 \\
\hline $\mathrm{Fe}_{2}$ & 5 & -0.007 & -0.010 & -0.021 & -0.032 & 0.002 & -0.028 & -0.023 & 0.006 & -0.034 \\
\hline $\mathrm{P}_{1}$ & 4 & -0.011 & 0.007 & 0.017 & -0.005 & 0.009 & 0.026 & 0.004 & 0.004 & 0.028 \\
\hline $\mathrm{P}_{2}$ & 4 & -0.006 & 0.011 & -0.007 & -0.001 & 0.012 & -0.024 & 0.005 & 0.008 & -0.021 \\
\hline $\mathrm{O}_{1}$ & 3 & -0.001 & 0.022 & -0.018 & -0.003 & 0.006 & -0.023 & 0.004 & 0.010 & -0.024 \\
\hline $\mathrm{O}_{2}$ & 3 & -0.016 & -0.010 & 0.016 & -0.014 & 0.016 & 0.026 & -0.005 & 0.004 & 0.029 \\
\hline $\mathrm{O} 2_{1}$ & 3 & -0.008 & 0.026 & 0.032 & -0.031 & -0.081 & 0.043 & -0.021 & -0.071 & 0.040 \\
\hline $\mathrm{O} 2_{2}$ & 3 & -0.014 & 0.015 & -0.008 & 0.000 & 0.016 & -0.022 & 0.004 & 0.015 & -0.019 \\
\hline $\mathrm{O} 3_{1}$ & 2 & -0.035 & -0.011 & 0.027 & -0.161 & 0.079 & 0.079 & -0.175 & 0.101 & 0.091 \\
\hline $\mathrm{O}_{3}$ & 2 & -0.008 & 0.002 & 0.002 & 0.020 & 0.031 & -0.024 & 0.010 & 0.017 & -0.018 \\
\hline
\end{tabular}

略低, 可能与后者使用的拟合经验参数有关. 进一 步计算包覆石墨烯或嗍氮类石墨烯的 $\mathrm{LiFePO}_{4}$ 的表 面能为 0.185 和 $0.253 \mathrm{~J} \cdot \mathrm{m}^{-2}$, 表明包覆层可以降低表 面能, 有利于纳米颗粒的分散, 阻止团聚.

相对于六配位, 五配位的 $\mathrm{Fe}-\mathrm{O}$ 键相互作用能增 大, 键长缩短. 因此, 无包覆的 $\mathrm{LiFePO}_{4}(010)$ 表面相 对收缩, 平均 $\mathrm{Fe}-\mathrm{O}$ 键长由 $\mathrm{LiFePO}_{4}$ 晶体中的 0.218 $\mathrm{nm}$ 降为 $0.212 \mathrm{~nm}$. 同时, $\mathrm{P}$ 原子的配位数没有变化, 体相和表面的平均 $\mathrm{P}-\mathrm{O}$ 键长相同, 为 $0.156 \mathrm{~nm}$. 相 反, 在包覆石墨烯 (或硼氮类石墨烯) 后, $\mathrm{LiFePO}_{4}$ (010)表面原子和包覆层原子成键, $\mathrm{Fe}-\mathrm{C}$ 键长为 $0.212 \mathrm{~nm}$ (或 $0.215 \mathrm{~nm}$ ), 与晶体中 $\mathrm{Fe}-\mathrm{O}$ 键长相近, 说明 $\mathrm{Fe}$ 原子和 $\mathrm{C}$ 原子之间具有较强的相互作用(图 2). 包覆后, $\mathrm{LiFePO}_{4}(010)$ 表面平均 $\mathrm{Fe}-\mathrm{O}$ 键长增大 至 $0.223 \mathrm{~nm}$ (或 $0.231 \mathrm{~nm}$ ), 平均 $\mathrm{P}-\mathrm{O}$ 键长增大至
$0.174 \mathrm{~nm}$ (或 $0.179 \mathrm{~nm}$ ). 上述结果表明, 包覆石墨烯 或嗍氮类石墨烯可以抵消 $\mathrm{LiFePO}_{4}$ 的表面收缩, 有 利于 $\mathrm{Li}^{+}$的迁入和迁出. 特别是, 裸露在外表面的 $\mathrm{O} 3$ 受包覆层影响, 平均位移超过 $0.1 \mathrm{~nm}$. 如果不计这些 具有大位移的表面氧原子, 则石墨烯和硼氮类石墨 烯包覆的 $\mathrm{LiFePO}_{4}(010)$ 表面的 $\mathrm{F}-\mathrm{O}$ 键平均键长分 别为 $0.215 \mathrm{~nm}$ 或 $0.218 \mathrm{~nm}$, 而 $\mathrm{P}-\mathrm{O}$ 键平均键长仍 为 $0.156 \mathrm{~nm}$, 变化不显著. 观察表面层原子在 $a 、 b 、 c$ 轴方向移动的距离(表2), 也可以发现无包覆的 $\mathrm{LiFePO}_{4}(010)$ 表面 $\mathrm{Fe}$ 原子和部分 $\mathrm{O}$ 原子沿 $b$ 轴收 缩, $\mathrm{Li}^{+}$通道也相应的发生收缩. 在包覆石墨烯或硼 氮类石墨烯后, 除了 $\mathrm{O} 2$ 原子外, 其它原子都沿 $b$ 轴 膨胀, 有利于 $\mathrm{Li}^{+}$的迁移. 由此可知, 包覆层不但引起 与其直接接触的 $\mathrm{LiFePO}_{4}$ 表面原子的相对位移, 也 影响其成键方式, 但对无直接接触原子的影响随距

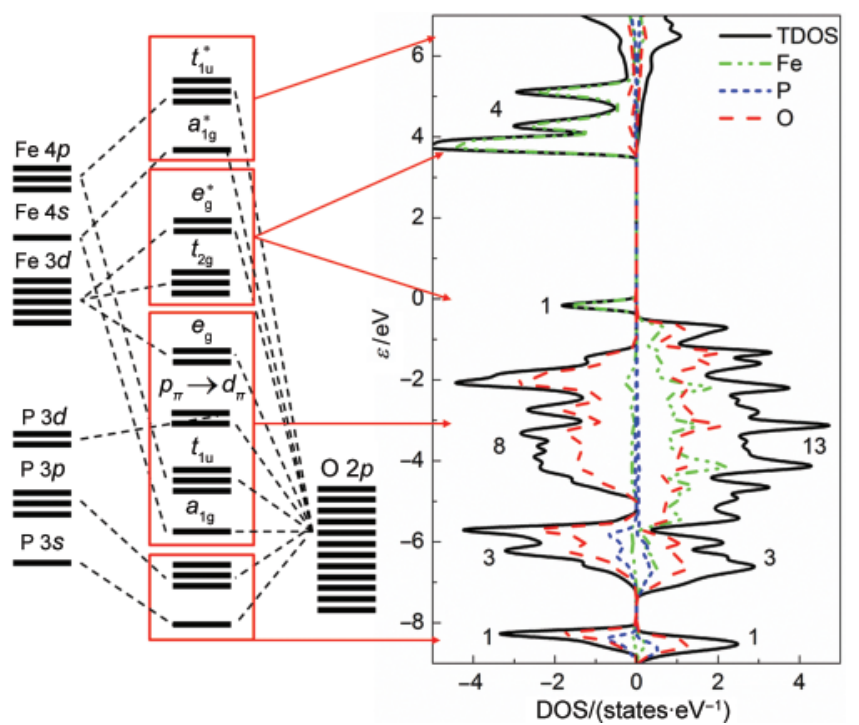

图 $3 \mathrm{LiFePO}_{4}$ 晶体的 $\mathrm{TDOS}$ 及其在原子轨道上的投影和布居分析

Fig.3 Total density of states (TDOS) and its projection on atomic orbits and occupation analysis of $\mathrm{LiFePO}_{4} \mathrm{crystal}$ The numbers beside the TDOS peaks are peak areas, representing the number of electrons. 
离的增大而降低.

\section{2 表面态密度}

\subsection{1 $\mathrm{LiFePO}_{4}$ 晶体的态密度}

$\mathrm{LiFePO}_{4}$ 晶体的总态密度(TDOS)显示价带顶和 导带底都是由自旋向下的 $\mathrm{Fe}$ 的 $3 d$ 轨道贡献(图 3). 受配位场影响, 电子成对能大于轨道分裂能, $\mathrm{Fe}$ 的 6 个 $3 d$ 电子 5 个自旋向上 1 个自旋向下, 排布为 $t_{2 g}^{4} e_{g}^{2}$, 有 4 个未成对电子. 在 $\mathrm{O} 2 p$ 带还有 8 对电子, 其中 $\mathrm{O}$ 的 6 对孤对电子填入 $\mathrm{Fe}$ 的 6 个成键轨道形成 $\mathrm{Fe}-\mathrm{O}$ 配位键, 2 对孤对电子与磷形成额外的两个 $d_{\pi}-p_{\pi}$ 配 位键. $\mathrm{P}$ 原子通过 $s p^{3}$ 杂化与 $\mathrm{O}$ 原子形成 $\mathrm{PO}_{4}$ 四面体, 其 $\sigma$ 键的能带出现在 $-9.0 \mathrm{eV}$ 到 $-5.0 \mathrm{eV}$ 之间.

\subsection{2 $\mathrm{LiFePO}_{4}$ 的(010)表面态密度}

无包覆的 $\mathrm{LiFePO}_{4}$ (010)表面的禁带宽度为 3.3 $\mathrm{eV}$, 略小于 $\mathrm{LiFePO}_{4}$ 晶体的 $3.7 \mathrm{eV}$, 说明即使是纯净 无包覆物的 $\mathrm{LiFePO}_{4}$, 减小 $\mathrm{LiFePO}_{4}$ 粒径, 增大比表 面积, 也可以改善 $\mathrm{LiFePO}_{4}$ 的导电性. 在 $\mathrm{LiFePO}_{4}$
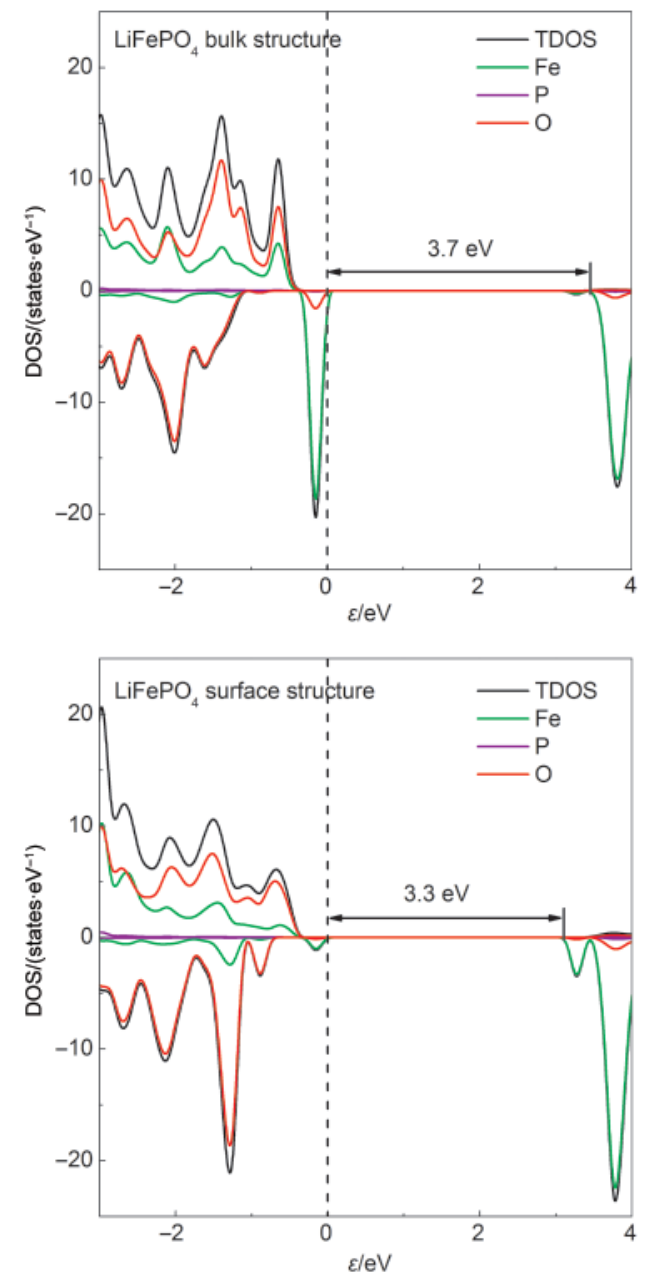

(010)表面上, $\mathrm{PO}_{4}$ 四面体结构完整, 只有 2 个 $\mathrm{Fe}-\mathrm{O}$ 键缺失; 相对于其它表面, (010)表面的键缺失最少, 表面结构稳定. 对比 $\mathrm{LiFePO}_{4}$ 晶体和 $\mathrm{LiFePO}_{4}$ 表面态 的 TDOS(图 4), 发现两者形状相似, 与文献报道一 致. ${ }^{30}$ 然而, 由于 $b$ 轴方向周期性缺失, 仍然可以观察 到 $\mathrm{LiFePO}_{4}$ 晶体和 $\mathrm{LiFePO}_{4}$ 表面态之间的两处不同: $\mathrm{LiFePO}_{4}(010)$ 表面 $\mathrm{Fe}-\mathrm{O}$ 键缺失, 表面氧 $\mathrm{O} 3$ 形成悬 空键, 电子能量升高, 使对应的 $-2.0 \mathrm{eV}$ 处的氧强峰 迁移至 $-1.3 \mathrm{eV}$ 处. 由于五配位的表面 $\mathrm{Fe}$ 原子的 $3 d$ 轨道和表面 $\mathrm{O}$ 原子未成键的 $2 p$ 轨道部分重合, 使得 出现于价带顶的 Fe $3 d$ 自旋向下电子由强峰降低为 弱峰, 同时在 $-1.3 \mathrm{eV}$ 处出现较强的 $\mathrm{Fe} 3 d$ 峰. 以上两 点表明, $\mathrm{LiFePO}_{4}(010)$ 表面性质活泼, 表面氧容易参 与成键.

相对于无包覆的 $\mathrm{LiFePO}_{4}(010)$ 表面 $3.3 \mathrm{eV}$ 的禁 带宽度, 包覆石墨烯后, 其禁带宽度下降了 $1.2 \mathrm{eV}$, 为 $2.1 \mathrm{eV}$. 表明石墨烯与 $\mathrm{LiFePO}_{4}$ 表面之间的相互作
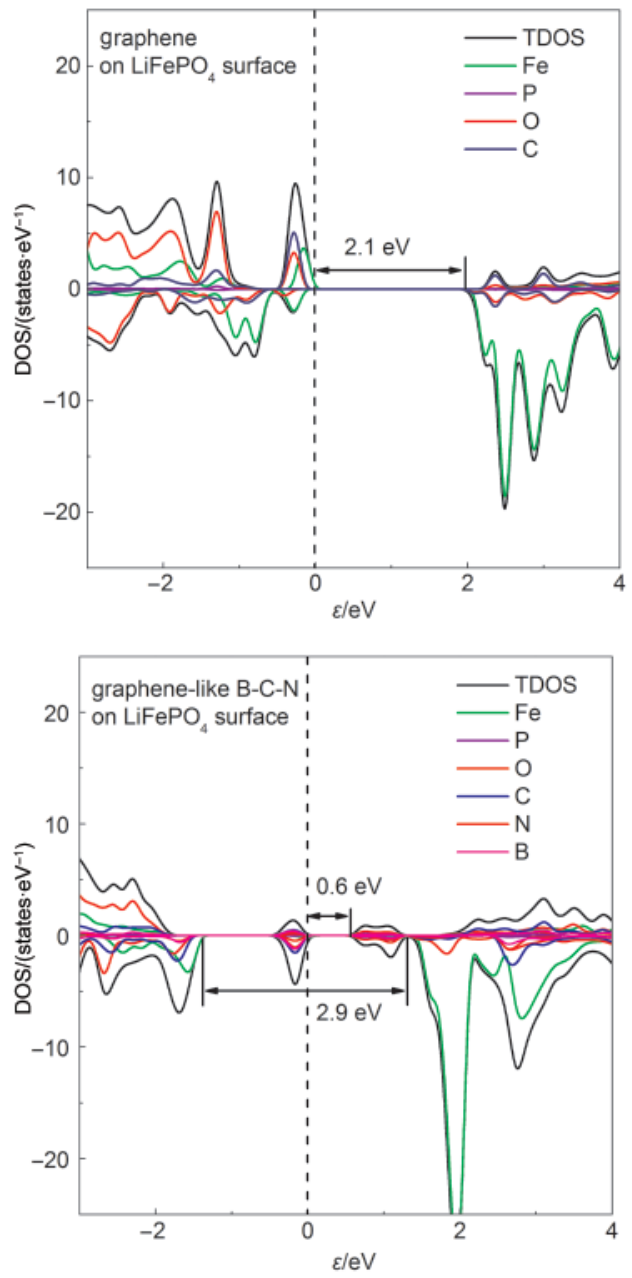

图 $4 \mathrm{LiFePO}_{4}$ 晶体、纯净的 $\mathrm{LiFePO}_{4}$ 、石墨烯包覆的 $\mathrm{LiFePO}_{4}$ 和类石墨烯 $\mathrm{B}-\mathrm{C}-\mathrm{N}$ 结构的 $\mathrm{LiFePO}_{4}$ 表面的 $\mathrm{TDOS}$

Fig.4 TDOS of bulk $\mathrm{LiFePO}_{4}$ crystal and these of pristine, and graphene or graphene-like B-C-N coated surface The vertical dashed lines represent the Fermi levels. 
用可以改变 $\mathrm{LiFePO}_{4}$ 表面的能带结构. 价带顶主要 由碳原子轨道贡献, 并和铁氧轨道能带相互作用, 使能量最高的电子离域于 $\mathrm{C} 2 p 、 \mathrm{Fe} 3 d$ 和 $\mathrm{O} 2 p$ 轨道. 包覆石墨烯后, TDOS 在 $\mathrm{O}$ 原子上的投影的最高峰 位置从 $-1.3 \mathrm{eV}$ 下降到 $-3.0 \mathrm{eV}$, 表明表面氧原子的 化学稳定性有所提高.

包覆硼氮类石墨烯后, $\mathrm{LiFePO}_{4}(010)$ 表面的能 带结构出现了显著变化. 虽然价带顶和导带底仍然 由 $\mathrm{Fe} 3 d$ 轨道贡献, 但由于硼氮类石墨烯和 $\mathrm{LiFePO}_{4}$ (010)表面氧相互作用, 禁带中出现两个带隙态小峰 (in-gap states), 间隔 $0.6 \mathrm{eV}$. 分析 DOS 在各原子上的 投影发现, 价带顶和导带底主要由嗍氮类石墨烯和 表面氧之间的成键作用形成. 带隙态的出现, 有利于 电子从带隙带跃迁至导带, 促进电子的传导(图 5).

对比石墨烯和硼氮类石墨烯的局域态密度发 现(图 6), 在费米面以下, 两者对 DOS 皆有贡献; 但 在费米面以上的带隙中, 石墨烯对 DOS 没有贡献, 嗍氮类石墨烯则有贡献, 更有利于电子的电导. $\mathrm{B}$ 原 子对 $\mathrm{LiFePO}_{4}$ 的表面有吸电子作用, 而 $\mathrm{N}$ 原子对 $\mathrm{LiFePO}_{4}$ 表面有给电子作用; 因此, 嗍氮类石墨烯和 $\mathrm{LiFePO}_{4}$ 表面间同时存在吸电子作用和给电子作 用. 在 $\mathrm{LiFePO}_{4}$ 颗粒放电时, 电子更容易从 $\mathrm{LiFePO}_{4}$ 表面迁移到嗍氮类石墨烯包覆层; 在 $\mathrm{LiFePO}_{4}$ 颗粒 充电时, 电子更容易从嗍氮类石墨烯包覆层迁移到 $\mathrm{LiFePO}_{4}$ 表面. 由此推断, 与石墨烯相比, 嗍氮类石 墨烯可以提高电子在 $\mathrm{LiFePO}_{4}$ 表面和包覆层之间的 迁移速率, 改善 $\mathrm{LiFePO}_{4}$ 表面与包覆层之间的导电 能力.

据文献报道, 表面包覆石墨烯可以使 $\mathrm{LiFePO}_{4}$

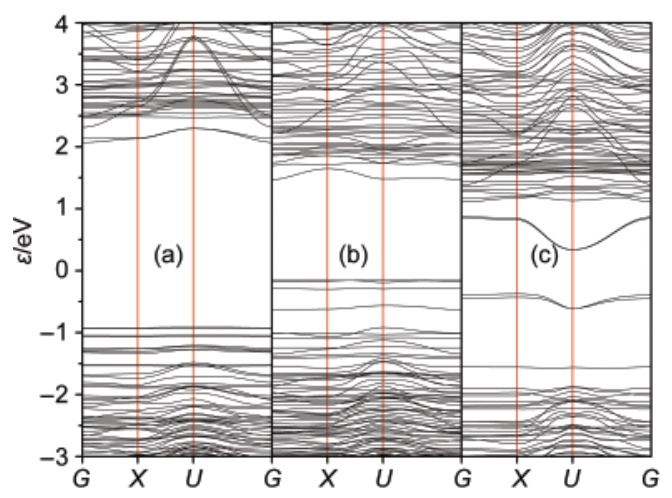

图 5 无包覆(a), 石墨烯片段包覆(b), 嗍氮类石墨烯片段包 覆(c)的 $\mathrm{LiFePO}_{4}(010)$ 表面态的能带结构

Fig.5 Surface band structures of pristine (a), graphene fragment (b), and graphene-like B-C-N fragment (c) coated $\mathrm{LiFePO}_{4}(010)$ surface

The vertical solid lines represent the Fermi levels.

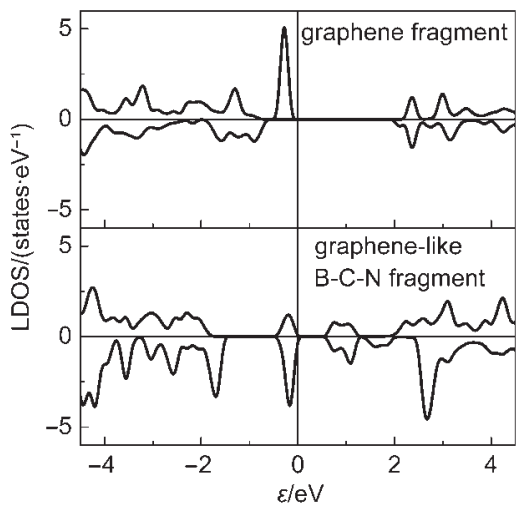

图 6 石墨烯片段和硼氮类石墨烯片段的局域态密度

Fig.6 Local density of states (LDOS) of graphene fragment and graphene-like B-C-N fragment

The vertical solid lines represent the Fermi levels.

的电导率提高 $10^{7}$ 倍; ${ }^{31,32}$ 同时, 相对于碳纳米管, 表 面包覆 $\mathrm{N}$ 掺杂碳纳米管的 $\mathrm{LiFePO}_{4}$ 具有更高的电导 率和充放电容量. ${ }^{33}$ 目前, 尚无文献报道在 $\mathrm{LiFePO}_{4}$ 表面包覆硼氮类石墨烯, 但根据本文计算, 包覆硼 氮类石墨烯将比包覆石墨烯等在提高磷酸铁锂材 料的导电性能方面具有更大的作用.

\section{4 结 论}

利用 DFT 分别研究无包覆、石墨烯包覆、嗍氮 类石墨烯包覆三种不同的 $\mathrm{LiFePO}_{4}(010)$ 表面的结 构及其态密度, 结果表明: 包覆层与 $\mathrm{LiFePO}_{4}$ 表面的 $\mathrm{P} 、 \mathrm{Fe}$ 和 $\mathrm{O}$ 原子之间皆有成键作用, 抵消了无包覆 $\mathrm{LiFePO}_{4}(010)$ 表面的收缩; 被石墨烯或嗍氮类石墨 烯包覆的 $\mathrm{LiFePO}_{4}(010)$ 表面的 $\mathrm{Fe}-\mathrm{O}$ 键平均键长比 无包覆表面的对应键长更接近于晶体内部, 使 $\mathrm{Li}^{+}$通 道扩张, 有利于 $\mathrm{Li}^{+}$的迁入和迁出.

计算的态密度表明: 相对于无包覆的 $\mathrm{LiFePO}_{4}$ (010)表面, 石墨烯包覆后 $\mathrm{LiFePO}_{4}$ (010)表面的禁带 宽度降低了 $1.2 \mathrm{eV}$, 有利于改善 $\mathrm{LiFePO}_{4}(010)$ 表面 电导性能. 特别是嗍氮类石墨烯包覆 $\mathrm{LiFePO}_{4}(010)$ 表面后, 禁带中出现两个带隙态, 间隔 $0.6 \mathrm{eV}$, 更利 于改善 $\mathrm{LiFePO}_{4}(010)$ 表面的电导性能.

\section{References}

(1) Padhi, A. K.; Nanjundaswamy, K. S.; Goodenough, J. B. J. Electrochem. Soc. 1997, 144 (4), 1188. doi: 10.1149/ 1.1837571

(2) Delacourt, C.; Laffont, L.; Bouchet, R.; Wurm, C.; Leriche, J. B.; Morcrette, M.; Tarascon, J. M.; Masquelier, C. J. Electrochem. Soc. 2005, 152 (5), A913.

(3) Chu, D. B.; Li, Y.; Song, Q.; Zhou, Y. Acta Phys. -Chim. Sin. 
2011, 27 (8), 1863. [褚道葆, 李 艳, 宋 奇, 周 莹. 物理化 学学报, 2011, 27 (8), 1863.] doi: 10.3866/PKU.WHXB 20110807

(4) Yu, H. M.; Zheng, W.; Cao, G. S.; Zhao, X. B. Acta Phys. -Chim. Sin. 2009, 25 (11), 2186. [余红明, 郑 威, 曹高劭, 赵新兵. 物理化学学报, 2009, 25 (11), 2186.] doi: 10.3866/PKU. WHXB20091113

(5) Ravet, N.; Goodenough, J. B.; Besner, S.; Simoneau, M.; Hovington, P.; Armand, M. Proceedings of the 196th ECS Meeting, Honolulu, Oct. 1999; pp 17-22.

(6) Xu, K.; Shen, L. F.; Mi, C. H.; Zhang, X. G. Acta Phys. -Chim. $\operatorname{Sin}$. 2012, 28 (1), 105. [徐科, 申来法, 米常焕, 张校刚. 物理化学学报, 2012, 28 (1), 105.] doi: 10.3866/PKU. WHXB201228105

(7) Sun, C. W.; Rajasekhara, S.; Goodenough, J. B.; Zhou, F. J. Am. Chem. Soc. 2011, 133 (7), 2132. doi: 10.1021/ja1110464

(8) Lepage, D.; Michot, C.; Liang, G. X.; Gauthier, M.; Schougaard, S. B. Angew. Chem. Int. Edit. 2011, 50 (30), 6884. doi: 10.1002/ anie. 201101661

(9) Chen, Z. H.; Dahn, J. R. J. Electrochem. Soc. 2002, 149 (9), A1184.

(10) Yuan, L. X.; Wang, Z. H.; Zhang, W. X.; Hu, X. L.; Chen, J. T.; Huang, Y. H.; Goodenough, J. B. Energy Environ. Sci. 2011, 4 (2), 269. doi: 10.1039/c0ee00029a

(11) Ouyang, C. Y.; Shi, S. Q.; Wang, Z. X.; Huang, X. J.; Chen, L. Q. Phys. Rev. B 2004, 69 (10), 104303. doi: 10.1103/PhysRevB. 69.104303

(12) Nishimura, S.; Kobayashi, G.; Ohoyama, K.; Kanno, R.; Yashima, M.; Yamada, A. Nat. Mater. 2008, 7 (9), 707. doi: $10.1038 /$ nmat 2251

(13) Fisher, C. A. J.; Islam, M. S. J. Mater. Chem. 2008, 18 (11), 1209. doi: $10.1039 / \mathrm{b} 715935 \mathrm{~h}$

(14) Wang, L.; Zhou, F.; Meng, Y. S.; Ceder, G. Phys. Rev. B 2007, 76 (16), 165435. doi: 10.1103/PhysRevB.76.165435

(15) Yang, S. F.; Zavalij, P. Y.; Whittingham, M. S. Electrochem. Commun. 2001, 3 (9), 505. doi: 10.1016/S1388-2481(01) 00200-4

(16) Dokko, K.; Koizumi, S.; Kanamura, K. Chem. Lett. 2006, 35 (3), 338. doi: 10.1246/cl.2006.338
(17) Chen, G. Y.; Song, X. Y.; Richardson, T. J. Electrochem. SolidState Lett. 2006, 9 (6), A295.

(18) Zaghib, K.; Mauger, A.; Gendron, F.; Julien, C. M. Chem. Mater. 2008, 20 (2), 462. doi: 10.1021/cm7027993

(19) Kresse, G.; Furthmuller, J. Comput. Mater. Sci. 1996, 6 (1), 15. doi: 10.1016/0927-0256(96)00008-0

(20) Kresse, G.; Furthmuller, J. Phys. Rev. B 1996, 54 (16), 11169. doi: 10.1103/PhysRevB.54.11169

(21) Blöchl, P. E. Phys. Rev. B 1994, 50 (24), 17953. doi: 10.1103/ PhysRevB.50.17953

(22) Kresse, G.; Joubert, D. Phys. Rev. B 1999, 59 (3), 1758. doi: 10.1103/PhysRevB.59.1758

(23) Perdew, J. P.; Chevary, J. A.; Vosko, S. H.; Jackson, K. A.; Pederson, M. R.; Singh, D. J.; Fiolhais, C. Phys. Rev. B 1992, 46 (11), 6671. doi: 10.1103/PhysRevB.46.6671

(24) Vosko, S. H.; Wilk, L.; Nusair, M. Can. J. Phys. 1980, 58 (8), 1200. doi: $10.1139 / \mathrm{p} 80-159$

(25) Zhou, F.; Cococcioni, M.; Kang, K.; Ceder, G. Electrochem. Commun. 2004, 6 (11), 1144. doi: 10.1016/j.elecom.2004.09.007

(26) Monkhorst, H. J.; Pack, J. D. Phys. Rev. B 1976, 13 (12), 5188. doi: 10.1103/PhysRevB.13.5188

(27) Hoang, K.; Johannes, M. Chem. Mater. 2011, 23 (11), 3003. doi: $10.1021 / \mathrm{cm} 200725 \mathrm{j}$

(28) Maxisch, T.; Ceder, G. Phys. Rev. B 2006, 73 (17), 174112. doi: 10.1103/PhysRevB.73.174112

(29) Rousse, G.; Rodriguez-Carvajal, J.; Patoux, S.; Masquelier, C. Chem. Mater. 2003, 15 (21), 4082. doi: 10.1021/cm0300462

(30) Ouyang, X.; Lei, M.; Shi, S.; Luo, C.; Liu, D.; Jiang, D.; Ye, Z.; Lei, M. J. Alloy. Compd. 2009, 476 (1), 462. doi: 10.1016/ j.jallcom.2008.09.028

(31) Oh, S. W.; Huang, Z. D.; Zhang, B. A.; Yu, Y.; He, Y. B.; Kim, J. K. J. Mater. Chem. 2012, 22 (33), 17215. doi: 10.1039/c2jm $33615 \mathrm{~d}$

(32) Wang, B.; Wang, D. L.; Wang, Q. M.; Liu, T. F.; Guo, C. F.; Zhao, X. S. J. Mater. Chem. A 2013, l (1), 135. doi: 10.1039/ c2ta00106c

(33) Yang, J. L.; Wang, J. J.; Li, X. F.; Wang, D. N.; Liu, J.; Liang, G. X.; Gauthier, M.; Li, Y. L.; Geng, D. S.; Li, R. Y.; Sun, X. L. J. Mater. Chem. 2012, 22 (15), 7537. doi: 10.1039/c2jm30380a 